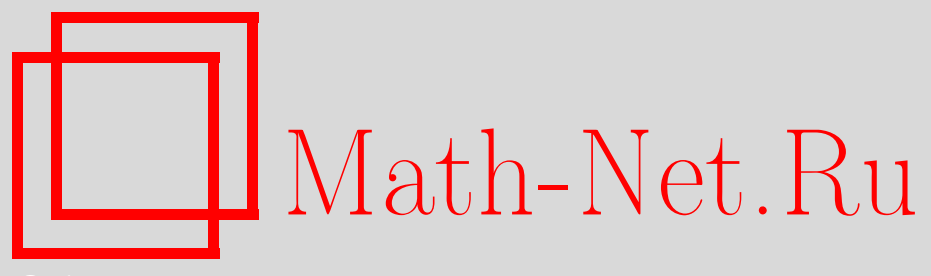

В. М. Бухштабер, Н. Ю. Ероховец, Алгебра операторов на кольце многогранников и квазисимметрические функции, УМН, 2010, том 65, выпуск 2, 197-198

DOI: https://doi.org/10.4213/rm9347

Использование Общероссийского математического портала Math-Net.Ru подразумевает, что вы прочитали и согласны с пользовательским соглашением http: //www. mathnet.ru/rus/agreement

Параметры загрузки:

IP : 54.89 .56 .158

26 апреля 2023 г., $17: 46: 36$

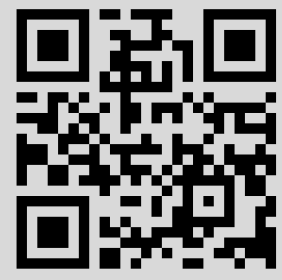




\section{Алгебра операторов на кольце многогранников и квазисимметрические функции}

\section{В. М. Бухштабер, Н. Ю. Ероховец}

Два выпуклых многогранника называются комбинаторно эквивалентными, если существует взаимно однозначное соответствие между их гранями, сохраняющее отношение включения.

Обозначим через $\mathscr{P}^{2 n}$ абелеву группу, порожденную всеми комбинаторными многогранниками размерности $n$. Тогда $\mathscr{P}^{2 n}=\bigoplus_{m \geqslant n+1} \mathscr{P}^{2 n, 2(m-n)}$, где $\mathscr{P}^{2 n, 2(m-n)}-$ конечно порожденная абелева группа, образующими которой являются $n$-мерные комбинаторные многогранники с $m$ гипергранями.

Декартово произведение многогранников $P \times Q$ задает в абелевой группе

$$
\mathscr{P}=\sum_{n \geqslant 0} \mathscr{P}^{2 n}=\mathscr{P}^{0}+\sum_{m \geqslant 2} \bigoplus_{n=1}^{m-1} \mathscr{P}^{2 n, 2(m-n)}
$$

структуру коммутативного ассоциативного кольца, в котором роль единицы играет точка pt. Многогранник называется неразложимым, если его нельзя представить в виде прямого произведения двух многогранников положительной размерности.

Лемма 1. Р является кольцом многочленов от неразложимых многогранников.

Для $k \geqslant 0$ определим оператор $d_{k}$, сопоставляющий многограннику $P^{n}$ сумму в кольце $\mathscr{P}$ всех его $(n-k)$-мерных граней $d_{k} P^{n}$.

ПРЕДЛОЖЕНИЕ $2 . d_{k}(P \times Q)=\sum_{i+j=k}\left(d_{i} P\right) \times\left(d_{j} Q\right)$.

В частности, оператор $d=d_{1}$ является дифференцированием.

Обозначим через $\mathscr{D}$ подкольцо в $\operatorname{Hom}_{\mathbb{Z}}(\mathscr{P}, \mathscr{P})$, порожденное операторами $d_{k}, k \geqslant 0$.

ОпредЕление 3. Алгеброй Лейбница-Хопфа над кольцом $A$ называется алгебра Хопфа $\mathscr{H}$ над $A$ с фиксированной конечной или счетной последовательностью образующих $H_{i}, i=1,2, \ldots$, и коумножением $\Delta H_{n}=\sum_{i+j=n} H_{i} \otimes H_{j}, H_{0}=1$.

Рассмотрим свободную алгебру Лейбница-Хопфа $\mathscr{Z}=\mathbb{Z}\left\langle Z_{1}, Z_{2}, \ldots\right\rangle$ со счетным числом образующих $Z_{i}$ (см. [1], [2]). Положим $\operatorname{deg} Z_{i}=2 i$ и $\Phi(t)=\sum_{i \geqslant 0} Z_{i} t^{i} \in \mathscr{Z}[[t]]$. Ясно, что $\mathscr{Z}$ является универсальной алгеброй Лейбница-Хопфа, т. е. для любой алгебры Лейбница-Хопфа $\mathscr{H}$ соответствие $Z_{i} \rightarrow H_{i}$ задает гомоморфизм алгебр Хопфа.

ОПредЕлЕниЕ 4. Обозначим через $\mathscr{U}$ алгебру Хопфа, универсальную в категории алгебр Лейбница-Хопфа $\mathscr{H}$ с антиподом $\chi\left(H_{i}\right)=(-1)^{i} H_{i}$.

ЛЕмма 5. $\mathscr{U} \simeq \mathscr{Z} / J_{\mathscr{U}}$, где двухсторонний идеал $J_{\mathscr{U}}$ порожден многочленами $u_{n}=\sum_{i=0}^{n}(-1)^{i} Z_{n-i} Z_{i}, n \geqslant 2$.

Многочлены $u_{n}$ - это коэффициенты ряда $\Phi(t) \Phi(-t)$, поэтому алгебра Хопфа $\mathscr{U}$ универсальна в категории алгебр Лейбница-Хопфа, для которых $\Phi(t) \Phi(-t)=1$.

Соответствие $Z_{k} \rightarrow d_{k}$ задает на кольце $\mathscr{P}$ структуру градуированного модуля Милнора (см. [3]) над алгеброй Хопфа $\mathscr{Z}$.

TEOpema 6. $\mathscr{D} \simeq \mathscr{Z} / J_{\mathscr{U}} \simeq \mathscr{U}$.

Кольцо простых многогранников $\mathscr{P}_{\mathrm{s}}$ (см. [4]) замкнуто относительно действия алгебры $\mathscr{D}$, при этом алгебра $\mathscr{D}\left(\mathscr{P}_{\mathrm{s}}\right)$ изоморфна алгебре разделенных степеней, т. е. $d_{k} d_{l}=\left(\begin{array}{c}k+l \\ k\end{array}\right) d_{k+l}$. 
Квазисимметрические функции были введены в работе [5]. Они образуют кольцо QSym $\subset \mathbb{Z}\left[t_{1}, t_{2}, \ldots\right], \operatorname{deg} t_{i}=2$, аддитивно порожденное элементарными квазисимметрическими мономами $M_{\left(j_{1}, \ldots, j_{k}\right)}=\sum_{l_{1}<\cdots<l_{k}} t_{l_{1}}^{j_{1}} \cdots t_{l_{k}}^{j_{k}}$. Это кольцо является кольцом многочленов над $\mathbb{Z}$ (см. [1]). QSym является алгеброй Хопфа, градуированно двойственной к алгебре $\mathscr{Z}$.

Обозначим через $\operatorname{QSym}(m)$ абелеву группу, порожденную всеми квазисимметрическими мономами от $m$ переменных.

Лемма 7. Многочлен $g \in \mathbb{Z}\left[t_{1}, \ldots, t_{m}\right]$ принадлежст $\mathrm{QSym}(m)$ тогда и толъко тогда, когда $g\left(0, t_{1}, \ldots, t_{m-1}\right)=g\left(t_{1}, 0, t_{2}, \ldots, t_{m-1}\right)=\cdots=g\left(t_{1}, \ldots, t_{m-1}, 0\right)$.

Уже из этой леммы вытекает, что $\mathrm{QSym}(m)$ является кольцом.

Пусть $S=\left\{a_{1}, \ldots, a_{k}\right\} \subset\{0,1, \ldots, n-1\}$. Положим $l(S)=k$. Флаговым числом $f_{S}$ называется число возрастающих последовательностей граней $F^{a_{1}} \subset F^{a_{2}} \subset \cdots \subset F^{a_{k}}$, $\operatorname{dim} F^{a_{i}}=a_{i}$. Структура модуля Милнора на кольце $\mathscr{P}$ индуцирует естественный кольцевой гомоморфизм $\mathscr{F}: \mathscr{P} \rightarrow \mathrm{QSym}[\alpha], \operatorname{deg} \alpha=2$ :

$$
\mathscr{F}_{\mathscr{P}}\left(P^{n}\right)=\sum_{k \geqslant 0} \sum_{l(S)=k} f_{S} \alpha^{a_{1}} M_{\omega(S)},
$$

где $\omega(S)=\left(n-a_{k}, a_{k}-a_{k-1}, \ldots, a_{2}-a_{1}\right)$. Заметим, что $\mathscr{F}_{\mathscr{P}}=\lim _{m} \mathscr{F}_{m}$, где $\mathscr{F}_{m}: \mathscr{P} \rightarrow$ $\operatorname{QSym}(m)[\alpha], \mathscr{F}_{m}(P)=\xi_{\alpha} \Phi\left(t_{m}\right) \cdots \Phi\left(t_{1}\right)(P)$, и $\xi_{\alpha}: \mathscr{P} \rightarrow \mathbb{Z}[\alpha]$ - кольцевой гомоморфизм такой, что $\xi_{\alpha} P^{n}=\alpha^{n}$.

Имеем $\mathscr{F}_{1}\left(P^{n}\right)=\xi_{\alpha} \Phi\left(t_{1}\right) P^{n}=\alpha^{n}+f_{n-1} \alpha^{n-1} t_{1}+\cdots+f_{0} t_{1}^{n}$ - однородный $f$-полином от двух переменных [4]. Таким образом, полином $\mathscr{F}$ есть обобщение $f$-полинома.

Отметим, что образ многогранника $P^{n}$ в QSym однозначно определяется любым из многочленов $\mathscr{F}_{m}\left(P^{n}\right), m \geqslant n$. Если $P^{n}-$ простой многогранник, то $\mathscr{F}\left(P^{n}\right)=$ $\mathscr{F}_{1}\left(\alpha, \sigma_{1}\right)$, где $\sigma_{1}=t_{1}+t_{2}+\cdots=M_{(1)}$.

Теорема 8. 1) $\mathscr{F}(\mathscr{P}) \otimes \mathbb{Q}$ является кольцом многочленов, $\operatorname{dim} \mathscr{F}\left(\mathscr{P}^{2 n}\right) \otimes \mathbb{Q}=c_{n}$, где $c_{n}-n$-е число Фибоначчи $\left(c_{0}=c_{1}=1, c_{n+1}=c_{n}+c_{n-1}, n \geqslant 1\right)$.

2) Группа $\mathscr{F}_{m}\left(\mathscr{P}^{2 n}\right) \subset \mathrm{QSym}(m)[\alpha], m \geqslant n$, coвпадает с группой однородных многочленов $\mathrm{g}$ степени $2 n$ таких, что

$$
\begin{aligned}
& g\left(\alpha, t_{1},-t_{1}, t_{3}, \ldots, t_{m}\right)=g\left(\alpha, 0,0, t_{3}, \ldots, t_{m}\right), \\
& g\left(\alpha, t_{1}, t_{2},-t_{2}, \ldots, t_{m}\right)=g\left(\alpha, t_{1}, 0,0, \ldots, t_{m}\right) \text {, }
\end{aligned}
$$

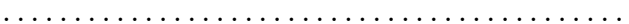

$$
\begin{aligned}
& g\left(\alpha, t_{1}, \ldots, t_{m-1},-t_{m-1}\right)=g\left(\alpha, t_{1}, \ldots, 0,0\right), \\
& g\left(-\alpha, t_{1}, \ldots, t_{m-1}, \alpha\right)=g\left(\alpha, t_{1}, \ldots, t_{m-1}, 0\right) .
\end{aligned}
$$

Эти уравнения равносильны соотношениям Байер-Биллера на флаговые числа многогранников (см. [6]).

\section{Список литературы}

[1] M. Hazewinkel, Adv. Math., 164:2 (2001), 283-300. [2] I. M. Gelfand, D. Krob, A. Lascoux, B. Leclerc, V.S. Retakh, J.-Y. Thibon, Adv. Math., 112:2 (1995), 218-348. [3] С. П. Новиков, УМН, 47:5 (1992), 189-190. [4] В. М. Бухштабер, Тр. МИАН, 263 (2008), 18-43. [5] I. M. Gessel, Combinatorics and algebra, Contemp. Math., 34, Amer. Math. Soc., Providence, RI, 1984, 289-317. [6] M. M. Bayer, L. J. Billera, Invent. Math., 79:1 (1985), 143-157.

\section{B. M. Бухштабер (V. M. Buchstaber)}

Математический институт им. В. А. Стеклова РАН

E-mail: buchstab@mi.ras.ru

\section{Н. Ю. Ероховец (N. Yu. Erokhovets)}

Московский государственный университет им. М. В. Ломоносова

E-mail: erochovetsn@hotmail.com
Представлено С. П. Новиковым Принято редколлегией 01.03 .2010 